 \\ National Institute of Standards and Technology U.S. Department of Commerce \\ NIST Technical Note 1551
}

\section{Recommended Terminology for Microwave Radiometry}

James Randa Janne Lahtinen Adriano Camps A. J. Gasiewski Martti Hallikainen David M. Le Vine Manuel Martin-Neira Jeff Piepmeier Philip W. Rosenkranz Christopher S. Ruf James Shiue Niels Skou 



\section{NIST Technical Note 1551 \\ Recommended Terminology for \\ Microwave Radiometry}

\author{
James Randa \\ Electromagnetics Division \\ Electronics and Electrical Engineering \\ Laboratory \\ National Institute of Standards and \\ Technology \\ Boulder, CO 80305-3328 \\ Janne Lahtinen \\ Harp Technologies Ltd. \\ 02150 Espoo, Finland

\section{Adriano Camps} \\ Department of Signal Theory and \\ Communications \\ Universitat Politecnica de Catalunya \\ Campus Nord \\ 08034 Barcelona, Spain
}

\section{A. J. Gasiewski}

NOAA-CU Center for Environmental

Technology (CET)

Univ. of Colorado at Boulder

Boulder, CO 80309

\author{
Martti Hallikainen \\ Laboratory of Space Technology \\ Helsinki Univ. of Technology \\ 02150 Espoo, Finland \\ David M. Le Vine \\ James Shiue \\ Jeff Piepmeier \\ NASA, Goddard Space Flight Center \\ Greenbelt, MD 20771 \\ Manuel Martin-Neira \\ European Space Agency \\ 2200 AG Noordwijk \\ The Netherlands \\ Philip W. Rosenkranz \\ Massachusetts Institute of Technology \\ Cambridge, MA 02139 \\ Christopher S. Ruf \\ Space Physics Research Laboratory \\ Univ. of Michigan \\ Ann Arbor, MI 48109 \\ Niels Skou \\ Technical University of Denmark \\ B348, DK2800 Lyngby, Denmark
}

August 2008

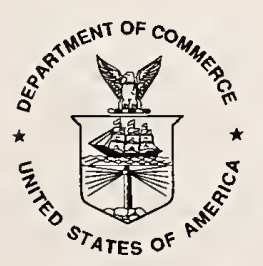

U.S. Department of Commerce

Carlos M. Gutierrez, Secretary

National Institute of Standards and Technology James M. Turner, Deputy Director 
Certain commercial entities, equipment, or materials may be identified in this document in order to describe an experimental procedure or concept adequately. Such identification is not intended to imply recommendation or endorsement by the National Institute of Standards and Technology, nor is it intended to imply that the entities, materials, or equipment are necessarily the best available for the purpose.

National Institute of Standards and Technology Technical Note 1551 Natl. Inst. Stand. Technol. Tech. Note 1551, 32 pages (August 2008) CODEN: NTNOEF 


\section{CONTENTS}

Page

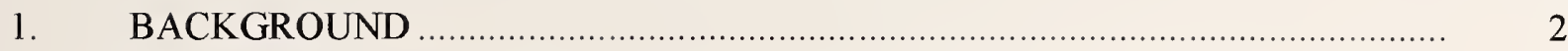

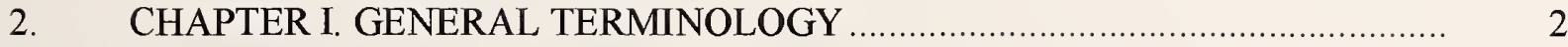

3. CHAPTER II. REAL-APERTURE RADIOMETERS …....................................... 6

4. CHAPTER III. POLARIMETRIC RADIOMETRY ……........................................ 13



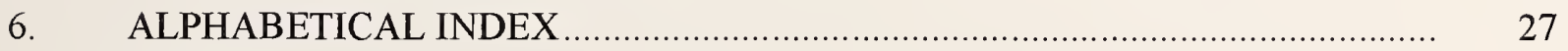





\title{
RECOMMENDED TERMINOLOGY FOR MICROWAVE RADIOMETRY
}

\author{
James Randa ${ }^{1}$, Janne Lahtinen ${ }^{2}$, Adriano Camps ${ }^{3}$, A. J. Gasiewski ${ }^{4}$, \\ Martti Hallikainen ${ }^{5}$, David M. Le Vine ${ }^{6}$, Manuel Martin-Neira ${ }^{7}$, Jeff Piepmeier ${ }^{6}$, \\ Philip W. Rosenkranz ${ }^{8}$, Christopher S. Ruf ${ }^{9}$, James Shiue ${ }^{6}$, and Niels Skou ${ }^{10}$ \\ ${ }^{1}$ Electromagnetics Division, NIST, Boulder, CO 80305 \\ ${ }^{2}$ Harp Technologies Ltd, 02150 Espoo, Finland \\ ${ }^{3}$ Department of Signal Theory and Communications, Universitat Politecnica de \\ Catalunya, Campus Nord, 08034 Barcelona, Spain \\ ${ }^{4}$ NOAA-CU Center for Environmental Technology (CET), Univ. of Colorado at \\ Boulder, Boulder, CO 80309 \\ ${ }^{5}$ Laboratory of Space Technology, Helsinki Univ. of Technology, 02150 Espoo, \\ Finland \\ ${ }^{6}$ NASA, Goddard Space Flight Center, Greenbelt, MD 20771 \\ ${ }^{7}$ European Space Agency, 2200 AG Noordwijk, The Netherlands \\ ${ }^{8}$ Massachusetts Institute of Technology, Cambridge, MA 02139 \\ ${ }^{9}$ Space Physics Research Laboratory, Univ. of Michigan, Ann Arbor, MI 48109 \\ ${ }^{10}$ Technical University of Denmark, B348, DK2800 Lyngby, Denmark
}

\begin{abstract}
We present recommended definitions for common terms in microwave remotesensing radiometry. Terms are grouped into three chapters: General Terminology, RealAperture Radiometers, and Polarimetric Radiometry. An alphabetical index lists the terms that are defined and the chapters in which the definitions are located.
\end{abstract}

Keywords: definitions, microwave radiometry, remote sensing, standard terminology 


\section{BACKGROUND}

Several years ago, a project was begun under the auspices of the Committee on Earth Observation Satellites (CEOS), Calibration and Validation Working Group, to compile standard definitions for terms used in microwave remote-sensing radiometry. A parallel effort was begun at about the same time in the NIST Electromagnetics Division, and the two efforts soon merged. A compilation of standard terminology and practices had been published for radiometry at optical frequencies [1], and had been found to be very useful. Since much of the terminology for microwave radiometry differs from that used at optical frequencies, a project was begun to develop a corresponding set of standard definitions for microwave radiometry. The effort was divided into four chapters, covering General Terminology (Chapter 1, or I), Real-Aperture Radiometers (II), Polarimetric Radiometry (III), and Interferometric Radiometry (IV). Recommendations and suggestions were solicited from the microwave radiometry community, and a website [2] and e-mail circulation list were established for I and II, to accept suggestions and to post the current definitions. After an initial burst of activity, the definitions for I and II stabilized, and they were presented to CEOS in 2003.

A "Radiometry Dictionary" page has now been added to the website of the IEEE Geoscience and Remote Sensing Society (GRSS) [3], under "Technical Resources." The purpose of the page is to increase the availability of the completed chapters, facilitate completion of the unfinished chapter, and raise awareness of the standard definitions. We expect that the list of standard definitions will evolve over time, with the completion of IV, as well as additions and modifications to I through III. The present paper contains the initial versions of I - III on the GRSS website. It thus constitutes a snapshot of I - III, documenting their state as of early 2008.

Many of the definitions have been drawn from other sources, in which case the source is referenced. Two of the most common sources, particularly for the terms in I, are the International Vocabulary of Basic and General Terms in Metrology (IVM) [4] and the Guide to the Expression of Uncertainty in Measurement (GUM) [5]. For terms contained in the IVM or GUM, we have tried to use those definitions. An alphabetical index follows the three chapters of definitions; it lists the terms defined and indicates in which chapter they are defined.

\section{CHAPTER I. GENERAL TERMINOLOGY}

Accuracy: closeness of the agreement between the result of a measurement and a true value of the measurand. Because the true value cannot be determined exactly, the measured or calculated value of highest available accuracy is typically taken to be the true value. If a value of higher accuracy than the value in question is not available, then the accuracy cannot be assigned a meaningful quantitative value.

Allan standard deviation (ASD): the square root of Allan variance. Both the ASD and the Allan variance can be used to describe different noise types and drift of the response 
of a radiometer receiver. Compared to Allan variance, however, the ASD has the advantage that the units are in kelvins.

Allan variance: a two-sample variance, where the variance is taken on the variable $y$. Each value of $y$ in a set has been averaged over an interval $\tau$ and the $y$ 's are taken in an adjacent series, i.e., no delay between the measurements of each:

$$
\sigma_{A}^{2}(\tau)=\frac{1}{2}\left\langle(\Delta y)^{2}\right\rangle
$$

where the brackets $<>$ denote the expectation value, and $\Delta y$ is the difference between sequential values of $y$. The Allan variance can be used to describe different noise types and drift of a radiometer receiver. See also: Allan Standard Deviation (ASD).

Bias (of a measuring instrument): systematic error of the indication of a measuring instrument. [4]

Note: The bias of a measuring instrument is normally estimated by averaging the error of indication over an appropriate number of repeated measurements.

Brightness: the radiance, the radiated power per unit solid angle per unit area normal to the direction defined by the solid angle $\Omega$.

Note: Brightness (of a surface) is often used for Spectral brightness density [6, 7]. The meaning is usually clear from the context.

Calibration: set of operations that establish, under specified conditions, the relationship between sets of values of quantities indicated by a measuring instrument or measuring system and the corresponding values realized by standards. The result of a calibration permits either the assignment of values of measurands to the indications or the determination of corrections with respect to the indications. [4]

Calibration equation (of a microwave radiometer): equation relating the primary measurand of the radiometer, e.g., the brightness temperature, to subsidiary measurands, such as powers, and to calibration quantities, such as standard values. (similar to [1])

Check standard: a standard that is used routinely to check measurements.

Drift: slow change of a metrological characteristic of a measuring instrument. [4]

Emissivity (of a surface): the spectral radiance of the surface relative to the spectral radiance of a perfect blackbody radiator at the same temperature, $e(\theta, \phi)=\frac{L_{f}(T, \theta, \phi)}{L_{f, B B}(T)}$.

Effective blackbody brightness temperature: the physical temperature of a perfect blackbody that would produce the same spectral brightness density or spectral radiance density as that under consideration. 
Error: result of a measurement minus a true value of the measurand. [4,5]

Notes: Since a true value cannot be determined, in practice a conventional true value is used. When it is necessary to distinguish "error" from "relative error," the former is sometimes called the "absolute error."

Experimental standard deviation: for a series of $n$ measurements of the same measurand, the quantity $s$ characterizing the dispersion of the results and given by the formula

$$
s=\sqrt{\frac{\sum_{i=1}^{n}\left(x_{i}-\bar{x}\right)^{2}}{n-1}},
$$

$x_{i}$ being the result of the $i$ th measurement and $\bar{x}$ being the arithmetic mean of the $n$ results considered. [4,5]

Notes: Considering the series of $n$ values as a sample of a distribution, $\bar{x}$ is an unbiased estimate of the mean $\mu$, and $s^{2}$ is an unbiased estimate of the variance $\sigma^{2}$ of that distribution. The expression $s / \sqrt{n}$ is an estimate of the standard deviation of the distribution of $\bar{x}$ and is called the experimental standard deviation of the mean.

Noise temperature (at a reference plane in a microwave circuit/network, $T_{n}$ ): the available noise power spectral density at the reference plane divided by Boltzmann's constant $\left(k_{B}\right)$; thus $\frac{d P_{n}^{a v}}{d f}=k_{B} T_{n}$ by definition, where the subscript $n$ denotes "noise." For a resistor at physical temperature $T_{p h y s,}$ the noise temperature is given by $k_{B} T_{n}=\frac{h f}{e^{\frac{h f}{k_{B} T_{p h y s}}}-1}$, so that for $\frac{h f}{k_{B} T_{\text {phys }}}<1, T_{n} \approx T_{\text {phys }}$.

Quantization level: in the quantization process, the discrete value assigned to a particular subrange of the analog signal being quantized. (adapted from [8])

Radiance $(L)$ : the radiated power per unit solid angle per unit area normal to the direction defined by the solid angle $\Omega$,

$$
L=\frac{d P}{d \Omega d A_{\perp}}
$$

where $d A_{\perp}=\cos \theta^{\prime} d A$, and $\theta^{\prime}$ is the angle between the direction defined by the solid angle and the normal to the area element $d A$.

Random error: result of a measurement minus the mean that would result from an infinite number of measurements of the same measurand carried out under repeatability conditions. [4,5]

Notes: Random error is equal to error minus systematic error. Because only a finite number of measurements can be made, it is possible to determine only an estimate of random error.

Reference temperature $\left(T_{0}\right)$ : temperature used in ratios to set a scale, conventionally taken as $T_{0}=290 \mathrm{~K}$. 
Relative error: error of measurement divided by a (conventional) true value of the measurand. $[4,5]$

Relative uncertainty: uncertainty of the measurand divided by the nominal value of that measurand.

Repeatability (of results of measurements): closeness of the agreement between the results of successive measurements of the same measurand carried out under the same conditions of measurement. [4]

Notes: Repeatability conditions include: the same measurement procedure, the same observer, the same measuring instrument used under the same conditions, the same location, and repetition over a short period of time. Repeatability may be expressed quantitatively in terms of the dispersion characteristics of the results.

Reproducibility (of results of measurements): closeness of the agreement between the results of measurements of the same measurand carried out under changed conditions of measurement. [4]

Notes: A valid statement of reproducibility requires specification of the conditions changed. Reproducibility may be expressed quantitatively in terms of the dispersion characteristics of the results.

Resolution: smallest difference between values of a measurand that can be meaningfully distinguished. (modified [4])

Response time: time interval between the instant when a stimulus is subjected to a specified abrupt change and the instant when the response reaches and remains within specified limits around its final steady value. [4]

Sensitivity: change in the response of a measuring instrument divided by the corresponding change in the stimulus. [4]

Spectral brightness density: the brightness per unit frequency, the spectral radiance density.

Spectral radiance density $\left(L_{f}\right):$ the radiance per unit frequency interval, $L_{f} \equiv \frac{d L}{d f}$

Stability: ability of a measuring instrument to maintain constant its metrological characteristics with time. [4]

Notes: Stability may be quantified in several ways, for example: in terms of the time over which a metrological characteristic changes by a stated amount, or in terms of the change in a characteristic over a stated time.

Systematic error: mean that would result from an infinite number of measurements of the same measurand carried out under repeatability conditions minus a true value of the measurand. $[4,5]$ 
Notes: Systematic error is equal to error minus random error. Like true value, systematic error and its causes cannot be completely known. For a measuring instrument, see "bias."

Traceability: property of the result of a measurement or the value of a standard whereby it can be related to stated references, usually national or international standards, through an unbroken chain of comparisons, all having stated uncertainties. [4]

True value: value consistent with the definition of a given particular quantity. $[4,5]$

Notes: This is a value that would be obtained by a perfect measurement. True values are by nature indeterminate.

Type-A uncertainty: an uncertainty or component of uncertainty that is evaluated by the statistical analysis of series of observations. (adapted from [5])

Type-B uncertainty: an uncertainty or component of uncertainty that is evaluated by means other than the statistical analysis of series of observations. (adapted from [5])

Uncertainty budget: a detailed tabulation of the sources of uncertainty and their respective contributions to the standard uncertainty in the measurand of interest; sometimes referred to as "error budget."

Uncertainty of measurement: parameter, associated with the result of a measurement, that characterizes the dispersion of the values that could reasonably be attributed to the measurand; also a measure of the possible error in the estimated value of the measurand as provided by the result of a measurement. See [5] or [9] for details.

Validation: the process of assessing, by independent means, the quality of the data products derived from the system inputs.

Note: In a remote-sensing application, validation is commonly performed by comparing the value of a higher-order data product (e.g. sea surface temperature) inferred from the measured value of the brightness temperature, with a direct measurement of that quantity.

Verification: the testing and analysis necessary to provide confirmation that all (e.g., instrument) requirements have been met.

\section{CHAPTER II. REAL-APERTURE RADIOMETERS}

Absolute accuracy: commonly used to denote the total uncertainty, excluding the radiometric resolution. When the radiometric resolution is evaluated by statistical means (type-A uncertainty) and all other uncertainties are estimated by other means, the absolute accuracy corresponds to the total type-B uncertainty.

Angular resolution: angular width of antenna beam, usually defined as the half-power beam width. See also antenna beam width. 
Antenna aperture temperature $\left(T_{A, a p}\right)$ : the temperature of an ideal black body that would result in the same received power at the antenna aperture as in the actual case. In the Rayleigh-Jeans approximation, it is equal to the average incident brightness temperature weighted by the antenna directivity pattern,

$$
T_{A, a p}\left(\Omega_{0}\right)=\frac{1}{4 \pi} \iint_{4 \pi} D\left(\Omega-\Omega_{0}\right) T_{b}(\Omega) d \Omega .
$$

The antenna aperture temperature corresponds to the antenna temperature at the aperture of the antenna.

Antenna beam width: the (full) angle at which the antenna's radiation pattern (in power units) is at half its maximum value. It is also known as the "Half-power full width" (HPFW), or simply the "Half-power beam width," (HPBW). In engineering convention, it is also known as the " $3 \mathrm{~dB}$ beam width."

The antenna radiation pattern depends on the specific plane of cut, and two principal planes are generally given. For a linearly polarized antenna they are the E-plane (the plane containing the radiation maximum and the E-field) and the H-plane (the plane containing the radiation maximum and the $\mathrm{H}$-field, which plane is perpendicular to the $\mathrm{E}$ plane).

Antenna gain $(G(\theta, \varphi))$ : the ratio of the radiation intensity in a given direction, to the radiation intensity that would be obtained if the antenna pattern were isotropic. (adapted from [10])

Note: This is equivalent to the commonly used definition that the antenna gain is the product of the directivity and the antenna ohmic efficiency, or radiation efficiency. If the direction is not specified, the direction of maximum radiation is implied.

Antenna ohmic efficiency $\left(\eta_{\Omega}\right)$ : the ratio of the total radiated power divided by the total power accepted by the antenna. It is also equivalent to the ratio of the antenna radiation resistance $\left(R_{r a d}\right)$ divided by the sum of the antenna radiation resistance and the antenna ohmic resistance $\left(R_{\Omega}\right)$ :

$$
\eta_{\Omega}=\frac{P_{r a d}}{P_{i n}}=\frac{R_{r a d}}{R_{r a d}+R_{\Omega}} .
$$

It is also commonly called the radiation efficiency [11].

Antenna output temperature $\left(T_{A, o u t}\right)$ : the physical temperature of a matched impedance that delivers to the receiver the same noise power as the antenna. It includes two terms: the noise coming from the environment attenuated by the antenna ohmic efficiency and the thermal noise added by the antenna ohmic losses. In the Rayleigh-Jeans approximation,

$$
T_{A, \text { out }}\left(\Omega_{0}\right)=\eta_{\Omega} T_{A, a p}\left(\Omega_{0}\right)+\left(1-\eta_{\Omega}\right) T_{p}
$$


where $T_{p}$ is the physical temperature of the antenna and $\eta_{\Omega}$ is the ohmic efficiency of the antenna. The antenna output temperature is related to the input noise temperature of the receiver $\left(T_{\text {rec,in }}\right)$ by

$$
T_{r e c, \text { in }}=\frac{h f}{e^{/ k T_{A, o u t}-1}}
$$

Antenna temperature $\left(T_{a}\right)$ : the temperature $(\mathrm{K})$ equivalent of the power received with an antenna. The distinction between the antenna temperature, $T_{a}$, and the brightness temperature, $T_{b}$, is as follows. The antenna temperature is the equivalent of $T_{b}$ after being spatially averaged by the direction-dependent antenna directivity $D(\Omega)$. To eliminate ambiguity, one should specify the point to which the calibrated antenna temperature is referenced. If no point is specified, it is assumed to be at the antenna aperture, in which case it corresponds to the antenna aperture temperature.

For example, in traditional radio astronomy this point is usually the output port of the antenna, because that is the point in the system where the calibration loads are situated. In this case, the antenna temperature corresponds to the antenna output temperature. In other systems, particularly those with small antennas, the calibration targets are outside the antenna. In such a case, the calibrated antenna temperature refers to a point outside the antenna aperture, and the ohmic losses of the antenna are irrelevant. In still other cases, the calibration targets may lie between a large main reflector and a smaller feed antenna. Then the calibration of antenna temperature would remove feed antenna losses but not those of the main reflector.

Blackbody: see perfect blackbody.

Blackbody load: a microwave load with characteristics very close to those of a perfect blackbody within a certain frequency range.

Boresight: the beam-maximum direction of a highly directive antenna. [6]

Brightness Temperature $\left(T_{b}\right)$ : the temperature $(\mathrm{K})$ equivalent, by the inverse Planck function, to a spectral radiance $\left(\mathrm{W} \cdot \mathrm{Hz}^{-1} \cdot \mathrm{m}^{-2} \cdot \mathrm{sr}^{-1}\right)$, emitted by a blackbody at temperature $T_{b}$.

In general, $T_{b}$ is a function of frequency and direction $\Omega$, where $\Omega$ stands for angles $\theta$ and $\phi$. Thus, for unpolarized radiation, $T_{b}$ is the temperature of a blackbody that would emit the same radiance at the specified frequency and direction. By extension, for polarized radiation, the brightness temperature of each polarization is the temperature of a blackbody that would emit the same radiance in the specified polarization at the specified frequency and direction. In the Rayleigh-Jeans limit, the microwave power per unit bandwidth received by a radiometer is $P=k T_{b}$, where $k$ is Boltzmann's constant.

Close-coupling: the technique by which the receiving aperture of a radiometer is coupled to a blackbody load so that the system is closed to stray radiation. 
Directivity (of antenna in a given direction $(D(\theta, \varphi))$ : the ratio of the radiation intensity in a given direction from the antenna to the radiation intensity averaged over all directions. [6]

Notes: The average radiation intensity is equal to the total power radiated by the antenna divided by $4 \pi$. If the direction is not specified, the direction of maximum radiation is implied.

Dwell time: time interval required for the antenna beam to scan across one beam width, at constant speed, along the scan direction.

Note: This and the definition of radiometric resolution per beamwidth assume one sample per footprint; if the radiometer samples at twice this rate, the integration time is halved and the radiometric resolution per beam width is increased by a factor of $\sqrt{2}$.

Effective Field of View (EFOV): When the radiometer antenna is scanning across the earth's surface while the radiometer integrates, then the static instantaneous field of view (IFOV, in length unit and along the scanning direction), which is based purely on geometrical projections of the antenna beam and the earth's surface, is no longer valid. Instead, the Scanned EFOV or ESFOV along the scan direction is used in its place. The ESFOV takes into account the scan-induced "smear" effect, and in general is slightly larger than the static IFOV. The extent of the smear depends on the length of the integration time, the shape of the original antenna beam, and the scan speed with time.

In order to be specific, the cross-track EFOV (EFOV-CT) is defined only for the case in which: (i) the scan speed is constant, and (ii) the integration time is equal to the time required to move the antenna beam one IFOV. Again CT implies the scanning direction, which may or may not be the cross-track direction.

As an example, the EFOV-CT of a Gaussian shaped beam is $25 \%$ larger than its IFOV, according to the above definition (i) and (ii).

The EFOV-CT is determined as follows:

First obtain the Exposure function $\mathrm{E}(\mathrm{x})$, which is the integral of the product of the antenna pattern $\mathrm{G}(\mathrm{x}-\mathrm{m})$ and the integration time $\Delta \mathrm{t}$,

$$
E(x)=\{G(x-m) \Delta t,
$$

where $m=v t$ is the coordinate of the antenna beam center, which is moving at a scan velocity of $v$. The integration is from $t=0$ to $t=\tau$.

Following the same half-power point principle, the ESFOV-CT is determined by obtaining the points $\mathrm{x} 1$ and $\mathrm{x} 2(\mathrm{x} 2>\mathrm{x} 1$ assumed) where the values of $\mathrm{E}(\mathrm{x})$ are half of its maximum value, and the ESFOV-CT value is equal to $\mathrm{x} 2-\mathrm{x} 1$.

Effective input noise temperature (of receiver, $T_{r e c}$ ): the input termination noise temperature which, when the input termination is connected to a noise-free equivalent of the receiver, would result in the same output noise power as that of the actual receiver connected to a noise-free input termination. [12] It is related to the receiver noise figure by 


$$
F_{\text {rec }}=\left(\frac{T_{r e c}+T_{0}}{T_{0}}\right),
$$

where $T_{0}=290 \mathrm{~K}$. It is a function of frequency, and it depends on the impedance or reflection coefficient of the input termination. It also depends on the location of the input reference plane; unless otherwise stated, the receiver noise temperature is assumed to be defined at the receiver input.

End-to-end calibration: calibration of the entire radiometer system as a unit, achieved by observing the values of output quantities (voltage, power, etc.) for known values of incident radiance at the antenna aperture.

External calibration: a calibration method that applies reference signals from targets that lie outside the radiometer. If these targets illuminate the antenna of the radiometer, an end-to-end calibration is obtained.

Half-power bandwidth $\left(B_{3 d B}\right)$ : the width at which the power response is half the maximum value; also called the $3-\mathrm{dB}$ bandwidth.

Instantaneous field of view (IFOV): the antenna beam width projected onto the earth's surface. See also static IFOV.

Since the antenna radiation pattern depends on the specific plane (containing the beam axis) of cut, so does the IFOV value. Conventionally, IFOVs at two principal planes are given: the E-plane (containing the E-vector of the equivalent dipole) and the H-plane (perpendicular to the E-plane). When a single IFOV value is used, it is the (arithmetic) mean of the IFOVs of the two principal planes.

Internal calibration: calibration of a radiometer by connecting embedded reference noise sources to the receiver chain; a common example is injection calibration, in which a noise diode is used as the embedded reference noise source.

Limiting radiometric resolution: the radiometric resolution evaluated for the case of zero input radiance or brightness temperature. Referred to as the "temperature sensitivity" in Radio Astronomy.

Noise-equivalent bandwidth $\left(B_{n}\right)$ : the width of an ideal rectangular filter (or other circuit or component) having the same maximum (available) gain as the real filter, and which produces the same output available power (integrated over frequency) as the real one when the input is white noise. In terms of the filter (available) gain $G$, $B_{n}=\frac{\int_{0}^{\infty} G(f) d f}{G_{\max }}$.

Noise figure (of receiver): at a specified input frequency, the ratio of 1 ) the total noise spectral power density at a corresponding output frequency available at the output port when the noise temperature of the input termination is $T_{0}=290 \mathrm{~K}$, to 2) that portion of 
the total output noise spectral power density that is engendered by the input termination. [12] The noise figure depends on the impedance or reflection coefficient of the input termination and on the location of the input reference plane. Unless otherwise stated, the receiver noise figure is assumed to be defined at the receiver input.

(Normalized) Antenna pattern $(t(\theta, \varphi))$ : the antenna gain (or directivity) in a specified direction divided by the maximum value of the antenna gain (or directivity). See antenna gain for the relationship between antenna gain and directivity.

Operating noise figure (of receiver): at a specified input frequency, the ratio of 1) the total noise spectral power density at a corresponding output frequency available at the output port when the noise temperature of the input termination is the actual input noise temperature (or brightness temperature, for a system), to 2) that portion of the total output noise spectral power density that is engendered by the input termination. (adapted from [13]) In terms of the effective input noise temperature, the operating noise figure is given by

$$
F_{O p}=\left(\frac{T_{r e c}+T_{i n}}{T_{i n}}\right),
$$

where $T_{\text {in }}$ is the nominal value of the input noise temperature.

Perfect blackbody: a perfect absorber (and therefore the best possible emitter) of thermal electromagnetic radiation, whose spectral radiance density (or spectral brightness density) is given by the Planck formula,

$$
L_{f}=\frac{2 h f^{3}}{c^{2}\left(e^{h f / k_{B} T}-1\right)},
$$

where $f$ is the frequency, $h$ is Planck's constant, $k_{B}$ is Boltzmann's constant, and $T$ is the physical temperature of the blackbody. (adapted from [7])

Pixel-to-pixel accuracy: the brightness temperature accuracy in measuring only one resolution element; the values of other resolution elements are not used for correction.

(Equivalent) Predetection bandwidth $\left(B_{p r e}\right)$ : the effective bandwidth of the predetection section of a radiometer, used in computing the ideal radiometer resolution.

In terms of the gain $G$ of the predetection section, $B_{p r e}=\frac{\left\{\int_{0}^{\infty} G(f) d f\right\}^{2}}{\int_{0}^{\infty} G^{2}(f) d f}$

Radiation efficiency: see antenna ohmic efficiency. [11]

Radiation intensity: the magnitude of the Poynting vector, i.e., the flux density of the radiation. Its dimensions are power per unit area. 
Radiometer: a very sensitive receiver, typically with an antenna input, that is used to measure radiated electromagnetic power.

Radiometric (or radiometer) resolution: the smallest change in input brightness temperature or radiance that can be detected in the system output. The radiometric resolution can be measured by computing the Type-A uncertainty, e.g., the standard deviation of the mean of a number of measurements of the same quantity made over a short enough time period that the system can be considered to be stable. It is also often estimated by using the ideal equation for a total-power radiometer, $\Delta T_{\min }=\frac{T_{S y s}}{\sqrt{B_{p r e} \tau}}$, or the variant of this equation that is appropriate for the particular radiometer in question.

Radiometric resolution per beam width: (for a continuously scanning radiometer) the radiometric sensitivity evaluated for an integration time equal to the dwell time.

Radiometric sensitivity: often used to mean radiometric resolution, but this use is discouraged in view of the definition of sensitivity.

Spatial resolution: the length of the major and/or minor axes diameters of the $3 \mathrm{~dB}$ contour of the antenna pattern projected onto the earth's surface. These two diameters may differ. See also IFOV.

Static IFOV: When the $I F O V$ is expressed in length units, it is used to express the diameter (arc length) of a circular area that is the intersection of the antenna beam (assumed to be a right circular cone) and the earth's surface (assumed to be a spherical shell). This is valid in the case of normal incidence.

When the radiometer antenna is viewing the earth at an oblique angle, then the intersection area (between the right circular cone of the antenna beam and the spherical earth surface) is a pear-shaped figure. In this case two arc lengths are used to represent the IFOV, a Major diameter or IFOV-Maj, and a Minor diameter or IFOV-Min.

Static IFOV-AT, static IFOV-CT: For a cross-track scanning beam, the words Minor and Major diameters can be replaced by Along-Track and Cross-Track, respectively. Here A-T and C-T also signify the directions parallel to, and perpendicular to the subsatellite track direction, respectively.

For a conical scanning beam, the terms A-T and C-T still apply.

System gain: There are three different definitions of power gain:

- Power gain: $G_{p}=\frac{P_{L}}{P_{i}}$,

- Transfer power gain: $G_{T}=\frac{P_{L}}{P_{a v G}}$, 
- Available power gain: $G_{a}=\frac{P_{a v L}}{P_{a v G}}$,

where $P_{L}$ is the power that is delivered to the load, $P_{i}$ is the power that is delivered by the source to the input port, and $P_{a v G}$ and $P_{a v L}$ are the available powers from the generator and to the load, respectively.

These three definitions of power gain are related by the following relationships:

$$
\begin{aligned}
& G_{T}=G_{p} M_{g}=G_{a} M_{L}, \\
& M_{g}=\frac{\left(1-\left|\Gamma_{i}\right|^{2}\right)\left(1-\left|\Gamma_{G}\right|^{2}\right)}{\left|1-\Gamma_{i} \Gamma_{G}\right|^{2}}, \\
& M_{L}=\frac{\left(1-\left|\Gamma_{o}\right|^{2}\right)\left(1-\left|\Gamma_{L}\right|^{2}\right)}{\left|1-\Gamma_{o} \Gamma_{L}\right|^{2}},
\end{aligned}
$$

and

$$
G_{p}=\frac{\left|S_{21}\right|^{2}\left(1-\left|\Gamma_{L}\right|^{2}\right)}{\left|1-S_{22} \Gamma_{L}\right|^{2}\left(1-\left|\Gamma_{i}\right|^{2}\right)},
$$

where $\Gamma_{i}, \Gamma_{o}, \Gamma_{G}$, and $\Gamma_{L}$ are respectively the receiver (amplifier) input (i) and output (o) reflection coefficients, and the source $(G)$ and load $(L)$ reflection coefficients. $M_{g}$ and $M_{L}$ are the mismatch factors [14] at the input $(g)$ and output $(L)$ reference planes.

We usually refer to $G_{a}$, since in practice the amplifiers, filters and other subsystems are never perfectly matched.

Tip-curve calibration (or tipping-curve calibration): $N$-point calibration ( $N \geq 2)$ using the atmosphere at different zenith angles as calibration target.

Two-point calibration: fixing the relationship between the input signal and the output response of a radiometer using two distinct input stimuli. Assuming a linear receiver, all possible input signal levels can now be retrieved from the radiometer output responses. In the case of an external end-to-end calibration, the input signal equals the antenna aperture temperature of the radiometer.

\section{CHAPTER III. POLARIMETRIC RADIOMETRY}

Note: In this chapter, the signals are assumed to be constant over frequency unless otherwise stated.

Adding analog correlator: a cross-correlator that measures additional Stokes parameters / modified Stokes parameters by post-detection summing of distinct principal 
polarizations as well as the squared sums of them. In a polarization frame defined by vertical polarization and horizontal polarization, for example, the third and fourth Stokes parameters / modified Stokes parameters are measured in the following way: The sum of the orthogonal waves is square detected, respectively, in phase and in quadrature, followed by the subtraction of orthogonal autocorrelations. Using Stokes parameters in brightness temperature / modified Stokes parameters in brightness temperature:

$$
\begin{aligned}
& T_{3}=T_{U}=\frac{\lambda^{2}}{k_{B} \cdot \eta \cdot B} \cdot\left[\left\langle\left|E_{v}+E_{h}\right|^{2}\right\rangle-\left\langle\left|E_{v}\right|^{2}\right\rangle-\left\langle\left|E_{h}\right|^{2}\right\rangle\right]=\frac{2 \cdot \lambda^{2}}{k_{B} \cdot \eta \cdot B} \cdot \operatorname{Re}\left\langle E_{v} E_{h}^{*}\right\rangle \\
& T_{4}=T_{V}=j \cdot \frac{\lambda^{2}}{k_{B} \cdot \eta \cdot B} \cdot\left[\left\langle\left|E_{v}+E_{h}\left(-\frac{\pi}{2}\right)\right|^{2}\right\rangle-\left\langle\left|E_{v}\right|^{2}\right\rangle-\left\langle\left|E_{h}\right|^{2}\right\rangle\right]=\frac{2 \cdot \lambda^{2}}{k_{B} \cdot \eta \cdot B} \cdot \operatorname{Im}\left\langle E_{v} E_{h}^{*}\right\rangle,
\end{aligned}
$$

where $\eta$ is the wave impedance of the medium, $E_{\mathrm{v}}$ is the vertical component of the electric field, and $E_{h}$ and $E_{h}\left(-\frac{\pi}{2}\right)$ are, respectively, the horizontal component of the electric field in phase and in quadrature with respect to the vertical one. Wavelength, the wave impedance of the medium, bandwidth, and Boltzmann's constant are denoted by $\lambda$, $\eta, B$, and $k_{\mathrm{B}}$, respectively. See also: coherent detection, direct correlating correlator.

Analog correlator: see analog cross-correlator.

Analog cross-correlator: a cross-correlator based on analog technology, i.e., the signals are not quantized prior to correlation. See also: adding analog correlator, coherent detection, digital cross-correlator, direct correlating correlator.

Calibration coefficients: elements of the gain matrix and the offset vector. See also: response of a fully polarimetric radiometer.

Channel (of a radiometer): a receiver chain that (in the ideal case) is sensitive to a certain Stokes parameter / modified Stokes parameter. See also: Stokes parameter mixing.

\section{Circular polarization: see polarization.}

Coherence matrix (sometimes called the coherency matrix): matrix representing the auto- and cross-correlation terms of two distinct polarizations. For example, based on vertical polarization and horizontal polarization, the coherence matrix is

$$
\bar{J}=\left[\begin{array}{cc}
\left\langle\left. E_{v}\right|^{2}\right\rangle & \left\langle E_{v} E_{h}^{*}\right\rangle \\
\left\langle E_{v}^{*} E_{h}\right\rangle & \left\langle\left|E_{h}\right|^{2}\right\rangle
\end{array}\right],
$$

where $E_{v}$ and $E_{h}$ are vertical and horizontal electric field components, respectively, and * denotes complex conjugate. 
More conveniently for radiometric applications, the coherence matrix can also be expressed in terms of brightness temperature, i.e. multiplying the above defined parameters by the factor $\lambda^{2} \cdot k_{\mathrm{B}}{ }^{-1} \cdot \eta^{-1} \cdot B^{-1}$, where $\lambda$ is the wavelength of the wave, $k_{\mathrm{B}}$ is Boltzmann's constant, $\eta$ is the wave impedance of the medium, and $B$ is the bandwidth:

$$
\overline{\bar{J}}=\left[\begin{array}{cc}
T_{v} & \frac{1}{2}\left(T_{3}+j T_{4}\right) \\
\frac{1}{2}\left(T_{3}-j T_{4}\right) & T_{h}
\end{array}\right] .
$$

Coherent detection: measurement of Stokes parameters / modified Stokes parameters by the cross-correlation of signals collected at principal polarizations; the technique requires the preservation of phase coherence between the signals that are correlated. Either adding analog correlator or directcorrelating correlator (analog or digital) can be used. In a polarization frame defined by two orthogonal linear polarizations, for example, the third and fourth Stokes parameters / modified Stokes parameters are measured by complex cross-correlation of waves at these polarizations. The first and second modified Stokes parameters are obtained by the autocorrelation of orthogonal signals; the retrieval of the first and second Stokes parameters requires additional post-detection summing. See also: direct correlating polarimetric radiometer.

Completely polarized wave: an electromagnetic wave with completely correlated orthogonal polarizations, i.e., the determinant of its coherence matrix is zero. Using Stokes parameters it can be written

$$
I^{2}=Q^{2}+U^{2}+V^{2}
$$

where $I, Q, U$, and $V$ are the first, second, third, and fourth Stokes parameters, respectively. Using modified Stokes parameters, on the other hand,

$$
\left(S_{v}+S_{h}\right)^{2}=\left(S_{v}-S_{h}\right)^{2}+S_{3}^{2}+S_{4}^{2}
$$

where $S_{v}, S_{h}, S_{3}$, and $S_{4}$ are the first, second, third, and fourth modified Stokes parameters, respectively.

See also: completely unpolarized wave, partially polarized wave.

Completely unpolarized wave: regardless of the polarization frame, the first and second modified Stokes parameters are equal (and the difference is thus zero) and the third and fourth Stokes parameters / modified Stokes parameters are zero. Using the terminology of Stokes parameters: $Q=U=V=0$. The determinant of its coherency matrix is equal to the wave's intensity. See also: completely polarized wave, partially polarized wave. 
Complex correlator: complex cross-correlator.

Complex cross-correlator: a device that estimates the time average of the product of the in-phase and quadrature components of two time-varying signals, $x(t)$ and $y(t)$. The inphase component of a signal, $I$, is its even, or Fourier cosine, component; and the quadrature component, $Q$, is its odd, or Fourier sine, component. If we use $x(t)$ to set the phase, the real part of the complex cross-correlation estimates $\left\langle I_{x} I_{y}\right\rangle$, and the imaginary part estimates $\left\langle I_{x} Q_{y}\right\rangle$. There are both digital and analog methods of implementing the complex correlation of band-limited signals, either while they are modulated by a carrier or at baseband.

Complex degree of coherence: the off-diagonal terms of the coherency matrix normalized by the geometric mean of the diagonal terms. Using vertical polarization and horizontal polarization, for example,

$$
\mu_{v h}=\frac{\left\langle E_{v} E_{h}^{*}\right\rangle}{\sqrt{\left\langle\left|E_{v}\right|^{2}\right\rangle\left\langle\left|E_{h}\right|^{2}\right\rangle}},
$$

where $E_{v}$ and $E_{h}$ are vertical and horizontal electric field components, respectively.

Correlator: see: cross-correlator.

Cross-correlator: a device that calculates the time average of the product of two distinct input signals.

Degradation factor: a quantity representing the degradation of the signal-to-noise ratio of polarimetric channels due to channel non-idealities:

$$
D=\frac{\left|\int_{0}^{\infty} H_{1}(f) H_{2}^{*}(f) d f\right|}{\sqrt{\left.\beta \cdot \int_{0}^{\infty}\left|H_{1}(f)^{2}\right| H_{2}^{*}(f)\right|^{2} d f}}
$$

where $H_{1}(f)$ and $H_{2}(f)$ are the complex voltage transfer functions of channels 1 and 2, respectively, prior to correlation, and $\beta$ is the width of the pass-band. Note that the degradation factor includes the fringe-washing effects generated by transfer function differences, but not those generated by propagation time differences. 
Degree of polarization: the ratio of completely polarized power to total power:

$$
d=\frac{\sqrt{\left(S_{v}-S_{h}\right)^{2}+S_{3}^{2}+S_{4}^{2}}}{S_{v}+S_{h}}=\frac{\sqrt{Q^{2}+U^{2}+V^{2}}}{I}
$$

where $S_{v}, S_{h}, S_{3}, S_{4}$ are the first, second, third, and fourth modified Stokes parameters, respectively, and $I, Q, U$, and $V$ are the first, second, third, and fourth Stokes parameters, respectively. See also: partially polarized wave.

Delay line: a microwave component used to generate time delay. In a correlating radiometer it is used to equalize the propagation times of distinct signals prior to crosscorrelation and reduce fringe-washing effects, or to introduce a specific delay in order to compute the cross-correlation at different time lags.

Digital correlator: see: digital cross-correlator.

Digital cross-correlator: a cross-correlator based on digital technology, i.e., the correlation of quantized signals is performed. Depending on the correlator configuration, two or more quantization levels are applied. See also: analog cross-correlator, coherent detection, direct correlating correlator.

Direct correlating correlator: an analog or digital device that directly estimates additional Stokes parameters / modified Stokes parameters by cross-correlation of the principal polarizations without any post-detection addition. Conventionally, the third and fourth Stokes parameters / modified Stokes parameters are estimated by correlating vertical polarization and horizontal polarization with each other. See also: adding analog correlator, digital cross-correlator, analog cross-correlator.

Direct correlating polarimetric radiometer: a polarimetric radiometer based on coherent detection and a direct correlating correlator. See also: polarization combining radiometer.

Fringe-washing effect: degradation in the cross-correlation of two non-zero bandwidth signals that can be caused by: 1) different transit times from the signal source to the antennas; and/or 2) different group delay responses (time delay at each frequency) of the receivers/correlators. The group delay response may include instrumental delays added in the receiving chain, and it is uniquely related to the frequency response through the following equation:

$$
\tau_{g}(\omega)=-\frac{d \Phi(\omega)}{d \omega}
$$

where $\omega$ is radian frequency and $\Phi(\omega)$ is the phase of the receiver's frequency response. 
Fully polarimetric calibration: the determination of the offset and gain matrix elements of a fully polarimetric radiometer. See also: response of a fully polarimetric radiometer, tri-polarimetric calibration.

Fully polarimetric calibration standard: a device for performing external calibration of polarimetric radiometers. It can generate at least four linearly independent polarized reference signals. Along with an unpolarized calibration reference, a fully polarimetric calibration standard enables the fully polarimetric calibration of fully polarimetric radiometers. See also: tri-polarimetric calibration standard.

Fully polarimetric radiometer: a radiometer capable of directly or indirectly measuring all four Stokes parameters / modified Stokes parameters.

Gain matrix: matrix that contains the amplification terms relating the observed Stokes parameters in brightness temperature / modified Stokes parameters in brightness temperature and the outputs of the radiometer channels. Diagonal elements of the matrix represent autocorrelation channel gains; off-diagonal elements represent crosspolarization terms generated by interchannel cross-talk and antenna cross-polarization. See also: offset vector, response of a fully polarimetric radiometer.

Horizontal brightness temperature: brightness temperature derived from field components in the direction of horizontal polarization. See also: vertical brightness temperature.

\section{Horizontal polarization: see polarization.}

Incoherent detection: measurement of Stokes parameters / modified Stokes parameters by post-detection summing of flux densities at distinct principal polarizations. Phase coherence among channels is not required. In a polarization frame defined by vertical polarization and horizontal polarization, for example, the third Stokes parameter / modified Stokes parameter can be obtained as a difference between flux densities at $+45^{\circ}$ and $-45^{\circ}$ skewed linear polarizations and the fourth Stokes parameter / modified Stokes parameter as a difference between flux densities at left-handed and right-handed circular polarizations. The first and second Stokes parameters are, respectively the sum and difference of the detected vertical and horizontal flux densities; the first and second modified Stokes parameters are, respectively, the detected vertical and horizontal flux densities. See also: coherent detection, polarization combining radiometer.

Inter-channel cross-talk: non-ideality of a radiometer receiver leading to signal leakage between channels. See also: Stokes parameter mixing.

Linear polarization: see: polarization.

Linearly polarized calibration standard: tri-polarimetric calibration standard, which is capable of generating reference signals at orthogonal linear polarizations and a linearly polarized reference signal. See also: fully polarimetric calibration standard. 
Modified Stokes parameters: the polarization of an electromagnetic wave is completely described by the four Stokes parameters. However, in remote sensing the so-called modified Stokes parameters are frequently used, where the first and second parameters represent, respectively, the flux density at vertical polarization and horizontal polarization. Using Stokes parameters, modified Stokes parameters are defined as:

$$
\begin{gathered}
S_{v}=\frac{I+Q}{2} \\
S_{h}=\frac{I-Q}{2} \\
S_{3}=S_{U} \\
S_{4}=S_{V},
\end{gathered}
$$

where $I, Q, U$, and $V$ are, respectively, the first, second, third, and fourth Stokes parameters. Note that in polarimetric remote-sensing radiometry, the modified Stokes parameters are conventionally expressed in terms of brightness temperature, i.e., modified Stokes parameters in brightness temperature.

Modified Stokes parameters in brightness temperature: modified Stokes parameters expressed in terms of brightness temperature (in kelvins):

$$
\begin{aligned}
& T_{v}=\frac{\lambda^{2}}{k_{B} \cdot B} \cdot S_{v} \\
& T_{h}=\frac{\lambda^{2}}{k_{B} \cdot B} \cdot S_{h} \\
& T_{3}=\frac{\lambda^{2}}{k_{B} \cdot B} \cdot S_{3} \\
& T_{4}=\frac{\lambda^{2}}{k_{B} \cdot B} \cdot S_{4},
\end{aligned}
$$

where $\lambda$ is the wavelength of the wave, $k_{\mathrm{B}}$ is Boltzmann's constant, and $B$ is the noiseequivalent bandwidth. The first, second, third and fourth modified Stokes parameters are denoted by $S_{v}, S_{h}, S_{3}$, and $S_{4}$, respectively. See also: modified Stokes parameters, modified Stokes vector in brightness temperature, Stokes parameters in brightness temperature.

Modified Stokes vector in brightness temperature: vector containing all four modified Stokes parameters in brightness temperature of a certain brightness temperature scene: 


$$
\bar{T}_{B, \bmod }=\left[\begin{array}{c}
T_{v} \\
T_{h} \\
T_{3} \\
T_{4}
\end{array}\right],
$$

where $T_{v}, T_{h}, T_{3}$, and $T_{4}$ denote the first, second, third and fourth modified Stokes parameters, respectively. See also: Stokes vector in brightness temperature.

Offset vector: vector that contains the responses of the radiometer channels associated with the zero input signal of each Stokes parameter. See also: gain matrix, response of a fully polarimetric radiometer.

Partially polarized wave: an electromagnetic wave consisting of completely polarized wave(s) and completely unpolarized wave(s), i.e., the degree of polarization is between 0 and 1 .

Phase modulation: an incoherent detection technique that enables the measurement of all Stokes parameters / modified Stokes parameters by a single-channel radiometer receiver. The phase modulating component can be installed prior to or after an antenna. In the former case a quasi-optical device based, e.g., on a phase retardation plate or a polarizing grid can be applied as a phase shifter.

Phase retardation plate: a quasi-optical microwave device that generates a phase shift between orthogonal linear polarizations passing through the plate. Special cases are a quarter-wave plate and a half-wave plate, which generate $\pi / 2$ and $\pi$ phase shifts, respectively. Retardation plates can be applied in fully polarimetric calibration standards to generate a circularly polarized signal from a linearly polarized signal, or in polarimetric microwave receivers to generate phase modulation.

Platform motion compensation: a procedure for compensating the changes of the observed Stokes parameters / modified Stokes parameters that are generated by the motion of the radiometer platform. These changes can be caused, e.g., by the rotation of the polarization frame of the antenna(s), which may lead to Stokes parameter mixing between the first three Stokes parameters / modified Stokes parameters, and variations in incidence angle.

Poincaré sphere: the polarization of any completely polarized electromagnetic wave can be expressed by two angles, which define the position on the surface of the so-called Poincaré sphere (see figure below). In this representation, left and right hand circular polarizations are on the North and South poles, respectively; and the four linear principal polarizations are located symmetrically on the equator. 


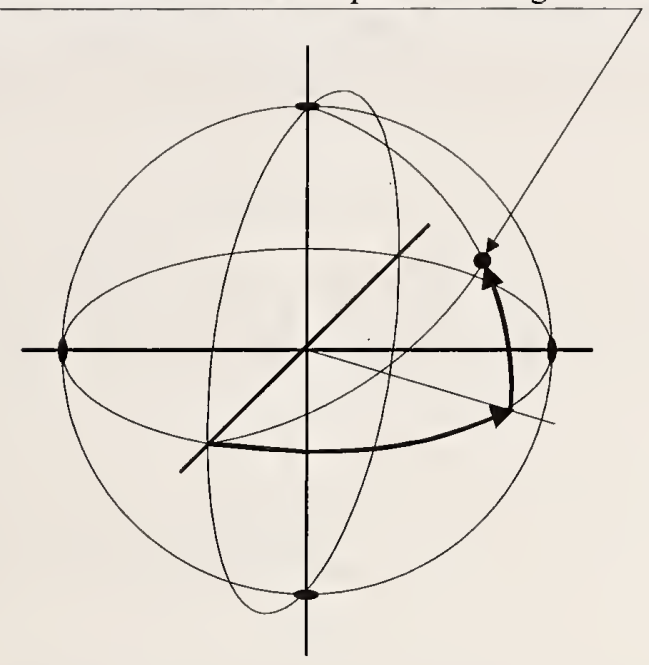

Polarimetric brightness temperatures: common name for the third and fourth Stokes parameters in brightness temperature / modified Stokes parameters in brightness temperature.

Polarimetric calibration: common name for tri-polarimetric calibration and fully polarimetric calibration.

Polarimetric calibration standard: common name for fully polarimetric calibration standards and tri-polarimetric calibration standards.

Polarimetric channels: common name for the radiometer channels that estimate the third and/or fourth Stokes parameters / modified Stokes parameters.

Polarimetric radiometer: a radiometer that measures additional Stokes parameters beyond the first and second Stokes parameters / modified Stokes parameters. Common name for tri-polarimetric radiometers and fully polarimetric radiometers.

Polarization: the direction of the electric $(E)$ or magnetic $(H)$ field in a propagating electromagnetic wave. A general, elliptically polarized electromagnetic plane wave propagating in the $\vec{r}$ direction can have its electric field expressed in phasor form as

$$
\vec{E}=\left(\hat{p} E_{p}+\hat{q} E_{q}\right) e^{-j k r}
$$

where $\hat{p}$ and $\hat{q}$ are unit vectors oriented perpendicular to $\vec{r}$ and satisfying $\hat{p} \times \hat{q}=\hat{r}=\vec{r} /|\vec{r}|, E_{p}$ and $E_{q}$ are the complex amplitudes of the electric field in the $\hat{p}$ and $\hat{q}$ directions, respectively, $k$ is the wavenumber of the propagating wave, and $r=|\vec{r}|$. The physical time-varying electric field is given by $\vec{E}(r, t)=\operatorname{Re}\left\{\vec{E} e^{j \omega t}\right\}$, where $\omega$ is the 
frequency in $\mathrm{rad} / \mathrm{s}$. There are a number of special cases of polarization that are commonly measured in radiometry:

Horizontal linear polarization satisfies: 1) $\hat{p}$ is oriented tangential to the earth's surface; 2) $\hat{q}$ has a positive projection onto the normal of the surface; 3) $E_{p} \neq 0$; and 4) $E_{q}=0$.

Vertical linear polarization satisfies: 1) $\hat{p}$ is oriented tangential to the earth's surface; 2) $\hat{q}$ has a positive projection onto the normal of the surface; 3) $E_{p}=0$; and 4) $E_{q} \neq 0$.

$+45^{\circ}$ slant linear polarization satisfies: 1) $\hat{p}$ is oriented tangential to the earth's surface; 2) $\hat{q}$ has a positive projection onto the normal of the surface; and 3 ) $E_{p}=E_{q}^{\prime} \neq 0$.

$-45^{\circ}$ slant linear polarization satisfies: 1) $\hat{p}$ is oriented tangential to the earth's surface; 2) $\hat{q}$ has a positive projection onto the normal of the surface; and 3 ) $E_{p}=-E_{q} \neq 0$.

Right hand circular polarization satisfies: 1) $E_{p} \neq 0$; and 2) $E_{q}=E_{p} e^{-j \pi / 2}$.

Left hand circular polarization satisfies: 1) $E_{p} \neq 0$; and 2) $E_{q}=E_{p} e^{j \pi / 2}$.

Polarization combining network: a microwave circuit that computes the sum or difference of two principal polarizations in-phase and/or quadrature to produce other principal polarizations. See also: incoherent detection, polarization combining radiometer.

Polarization combining radiometer: a polarimetric radiometer based on incoherent detection; post-detection summing of principal polarizations is applied to measure additional Stokes parameters / modified Stokes parameters. See also: direct correlating polarimetric radiometer.

Polarization frame: any orthogonal coordinate basis relating polarizations to each other.

Polarized brightness temperature scene: a scene that emits electromagnetic waves that are not completely randomly polarized. See also: degree of polarization, unpolarized brightness temperature scene.

Polarizing grid: a quasi-optical device that consists of parallel conductors. In the ideal case, in which the conductors have no ohmic losses and the grid is dense compared to the wavelength, a wave with electric field perpendicular to the conductors passes through the 
grid without attenuation, whereas a wave with electric field parallel to the conductors is completely reflected. In other orientations part of the wave is passed, part is reflected, and a cross-correlated (linearly polarized) wave component is generated. These characteristics can be applied to fabricate a linearly polarized calibration standard or a polarimetric radiometer based on phase modulation.

Principal polarizations: six polarizations by which an electromagnetic wave can be completely characterized at a certain frequency: four linear polarizations skewed in $45^{\circ}$ intervals $\left(0^{\circ}, 45^{\circ}, 90^{\circ} 135^{\circ}\right.$ rotated with respect to the reference polarization frame) and left- and right-handed circular polarizations. In remote sensing applications it is often convenient to select an orthogonal linear polarization frame with $0^{\circ}$ and $90^{\circ}$ aligned with horizontal polarization and vertical polarization, respectively. Then, $45^{\circ}$ and $135^{\circ}$ skewed linear polarizations can be referred to as $+45^{\circ}$ and $-45^{\circ}$ skewed linear polarizations, respectively.

Response of a fully polarimetric radiometer: the response of a radiometer is dependent on the brightness temperature scene and the characteristics of the radiometer. In terms of the modified Stokes vector in brightness temperature, this relationship for a fully polarimetric radiometer is

$$
\bar{r}_{\text {mod }}=\left[\begin{array}{l}
r_{v} \\
r_{h} \\
r_{3} \\
r_{4}
\end{array}\right]=\left[\begin{array}{llll}
g_{v v} & g_{v h} & g_{v 3} & g_{v 4} \\
g_{h v} & g_{h h} & g_{h 3} & g_{h 4} \\
g_{3 v} & g_{3 h} & g_{33} & g_{34} \\
g_{4 v} & g_{4 h} & g_{43} & g_{44}
\end{array}\right]\left[\begin{array}{c}
T_{v} \\
T_{h} \\
T_{3} \\
T_{4}
\end{array}\right]+\left[\begin{array}{c}
o_{v} \\
o_{h} \\
o_{3} \\
o_{4}
\end{array}\right]+\bar{n}_{\text {mod }}=\bar{g}_{\text {mod }} \bar{T}_{B, \bmod }+\bar{o}_{\text {mod }}+\bar{n}_{\text {mod }},
$$

where $\bar{r}_{\text {mod }}$ is the low frequency output response vector, $\bar{g}_{\text {mod }}$ is the gain matrix, $\bar{T}_{B}, \bmod$ is the modified Stokes vector in brightness temperature, $\bar{o}_{\bmod }$ is the offset vector, and $\bar{n}_{\text {mod }}$ is the zero-mean Gaussian instrument noise at the channel outputs. The off-diagonal terms of the gain-matrix represent interchannel cross-talk. In terms of the Stokes vector in brightness temperature, this relationship can be written as

$$
\bar{r}=\left[\begin{array}{l}
r_{I} \\
r_{Q} \\
r_{U} \\
r_{V}
\end{array}\right]=\left[\begin{array}{llll}
g_{I I} & g_{I Q} & g_{I U} & g_{I V} \\
g_{Q I} & g_{Q Q} & g_{Q U} & g_{Q V} \\
g_{U I} & g_{U Q} & g_{U U} & g_{U V} \\
g_{V I} & g_{V Q} & g_{V U} & g_{V V}
\end{array}\right]\left[\begin{array}{c}
T_{I} \\
T_{Q} \\
T_{U} \\
T_{V}
\end{array}\right]+\left[\begin{array}{c}
o_{I} \\
o_{Q} \\
o_{U} \\
o_{V}
\end{array}\right]+\bar{n}=\bar{g} \bar{T}_{B}+\bar{o}+\bar{n} .
$$

Sense of polarization: for an elliptical or circularly polarized field vector, the sense of rotation of the extremity of the field vector when its origin is fixed [6].

Stokes parameter mixing: contamination of a measured Stokes parameter / modified Stokes parameter value by other parameters, with the result that a certain radiometer channel is sensitive to more than one Stokes parameter / modified Stokes parameter. 
Stokes parameter mixing can be generated by, e.g., inter-channel cross-talk, antenna cross-polarization, and/or platform motion. See also: platform motion compensation, response of a fully polarimetric radiometer.

Stokes parameters: four parameters that completely characterize an electromagnetic wave at a certain frequency. The first Stokes parameter $(I)$ denotes the total Poynting vector or flux density of the wave. Expressing the other parameters in terms of linearly polarized components, the second Stokes parameter $(Q)$ is the difference between the flux densities polarized in two orthogonal directions in a given polarization frame, e.g., vertical polarization and horizontal polarization. The third $(U)$ and fourth $(V)$ Stokes parameters respectively represent twice the real and imaginary parts of the cross correlation between these orthogonal polarizations. The third Stokes parameter can also be defined as a difference between $+45^{\circ}$ and $-45^{\circ}$ skewed linearly polarized flux densities, and the fourth parameter as a difference between left-handed and right-handed circularly polarized flux densities:

$$
\begin{aligned}
& I=\frac{\left\langle\left|E_{v}\right|^{2}\right\rangle+\left\langle\left|E_{h}\right|^{2}\right\rangle}{\eta}=S_{v}+S_{h}, \\
& Q=\frac{\left\langle\left|E_{v}\right|^{2}\right\rangle-\left\langle\left|E_{h}\right|^{2}\right\rangle}{\eta}=S_{v}-S_{h}, \\
& U=\frac{2 \cdot \operatorname{Re}\left\langle E_{v} E_{h}^{*}\right\rangle}{\eta}=S_{+45}-S_{-45}, \\
& V=\frac{2 \cdot \operatorname{Im}\left\langle E_{v} E_{h}^{*}\right\rangle}{\eta}=S_{l c}-S_{r c},
\end{aligned}
$$

where $S$ is the flux density of the wave (in W. $\mathrm{m}^{-2}$ ). The subscripts $v, h,+45,-45, l c$, and $r c$ represent respectively the vertically, horizontally, $+45^{\circ}$ skewed linearly, $-45^{\circ}$ skewed linearly, left-handed circularly, and right-handed circularly polarized waves, $\eta$ is the wave impedance of the medium, and $E_{v}$ and $E_{h}$ are the electric field components with vertical and horizontal polarizations, respectively. Note that in polarimetric remote sensing radiometry the Stokes parameters are conventionally expressed in terms of brightness temperature, i.e., Stokes parameters in brightness temperature. See also: modified Stokes parameters, Stokes vector in brightness temperature.

Stokes parameters in brightness temperature: Stokes parameters expressed in terms of brightness temperature (in kelvins):

$$
\begin{aligned}
& T_{I}=\frac{\lambda^{2}}{k_{B} \cdot B} \cdot I, \\
& T_{Q}=\frac{\lambda^{2}}{k_{B} \cdot B} \cdot Q,
\end{aligned}
$$




$$
\begin{aligned}
& T_{U}=\frac{\lambda^{2}}{k_{B} \cdot B} \cdot U, \\
& T_{V}=\frac{\lambda^{2}}{k_{B} \cdot B} \cdot V,
\end{aligned}
$$

where wavelength, Boltzmann's constant, and noise-equivalent bandwidth are denoted by $\lambda, k_{\mathrm{B}}$, and $B$, respectively. The first, second, third and fourth Stokes parameters are denoted by $I, Q, U$, and $V$. Note that in the published literature on polarimetric remotesensing radiometry, $I, Q, U$, and $V$ have also been used for the Stokes parameters in brightness temperature (units kelvins). This practice may generate confusion and is therefore discouraged. See also: modified Stokes parameters in brightness temperature, Stokes vector in brightness temperature.

Stokes vector in brightness temperature: vector containing all four Stokes parameters in brightness temperature of a certain brightness temperature scene:

$$
\bar{T}_{B}=\left[\begin{array}{c}
T_{I} \\
T_{Q} \\
T_{U} \\
T_{V}
\end{array}\right],
$$

where $T_{I}, T_{Q}, T_{U}$, and $T_{V}$ denote the first, second, third and fourth Stokes parameters in brightness temperature, respectively. See also: modified Stokes vector in brightness temperature.

Tri-polarimetric calibration: the determination of the offset and gain terms (see: response of a fully polarimetric radiometer) of a tri-polarimetric radiometer. See also: fully polarimetric calibration.

Tri-polarimetric calibration standard: a device for external calibration of radiometers that can generate three linearly independent polarized brightness temperature scenes. Along with an unpolarized calibration standard, a tri-polarimetric calibration standard enables tri-polarimetric calibration. See also: fully polarimetric calibration standard.

Tri-polarimetric radiometer: a radiometer that measures three of the four Stokes parameters / modified Stokes parameters.

Unpolarized brightness temperature scene: a brightness temperature scene that emits completely unpolarized waves, i.e., the waves are completely randomly polarized.

Unpolarized calibration: a calibration procedure applying only unpolarized reference signals. Enables the determination of offset and diagonal gain terms (see: response of a fully polarimetric radiometer) of those radiometer channels, which are sensitive to unpolarized signals. See also: unpolarized reference standard. 
Unpolarized calibration standard: a calibration reference that generates unpolarized signals. See also: polarimetric calibration standard, unpolarized calibration.

Vertical brightness temperature: a brightness temperature derived from field components in the direction of vertical polarization. See also: horizontal brightness temperature.

Vertical polarization: see polarization.

We are grateful to Dr. Andreas Colliander of the European Space Agency and Professor Steven Reising of Colorado State University for very thorough and detailed reviews. This document is significantly better than it would otherwise have been due to their efforts. We also thank Dr. Paul Racette of NASA for early interest in and contributions to this effort.

\section{REFERENCES}

[1] C. Wyatt, V. Provalsky, and R. Datla, "Recommended practice; symbols, terms, units and uncertainty analysis for radiometric sensor calibration," Natl. Inst. Stand. Technol. Handbook 152 (1998 September).

[2] http://boulder.nist.gov/div818/stdterms/index.htm

[3] http://www.grss-ieee.org/

[4] International Vocabulary of Basic and General Terms, Second edition, International Organization for Standardization (ISO), Switzerland (1993).

[5] Guide to the Expression of Uncertainty in Measurement, International Organization for Standardization (ISO), Switzerland (1993).

[6] IEEE Standard Dictionary of Electrical and Electronics Terms, $4^{\text {th }}$ ed., ANSI/IEEE Std 100-1988.

[7] J.D. Krauss, Radio Astronomy, chapter 3, McGraw-Hill, New York (1966).

[8] Adapted from U.S. Federal Standard 1037C.

[9] B.N. Taylor and C.E. Kuyatt, "Guidelines for evaluating and expressing the uncertainty of NIST measurement results," Natl. Inst. Stand. Technol. Tech. Note 1297 (1994 September). 
[10] IEEE Standard 145-1993 (Standard Definitions of Terms for Antennas).

[11] D.M. Pozar, Microwave Engineering, chapter 12, John Wiley \& Sons, New York (1998).

[12] H.A. Haus et al., "IRE standards on methods of measuring noise in linear twoports, 1959," Proc. IRE, vol. 48, pp. $60-68$ (1960).

[13] H.A. Haus et al., "Description of the noise performance of amplifiers and receiving systems," Proc. IEEE, vol. 51, pp. 436 - 442 (1963).

[14] G.F. Engen, Microwave Circuit Theory and Foundations of Microwave Metrology, Ch. 3, Peter Perigrinus Ltd., London (1992).

\section{ALPHABETICAL INDEX}

Note: I, II, and III refer to chapters of the text. IVM and GUM indicate that the definition is given in the IVM [4] and/or GUM [5] and is not repeated in this document.

Absolute accuracy, II

Accuracy, I; see also Absolute accuracy, Pixel-to-pixel accuracy

Accuracy of a measuring instrument, I

Adding analog correlator, III

Allan standard deviation, I

Allan variance, I

Analog correlator, III

Analog cross correlator, III

Angular resolution, II

Antenna beam width, II

Antenna gain, II

Antenna ohmic efficiency, II

Antenna pattern, see (Normalized) antenna pattern

Antenna temperature, II

Antenna aperture temperature, II

Antenna output temperature, II

Bandwidth

Half-power bandwidth, II

Noise-equivalent bandwidth, II

(Equivalent) predetection bandwidth, II

Bias, I

Blackbody, II

Blackbody load, II

Boresight, II

Brightness, I

Brightness temperature, I; see also Effective black-body brightness temperature. 
Calibration, I

End-to-end calibration, II

External calibration, II

Internal calibration, II

Tip-curve calibration, II

Two-point calibration, II

Calibration coefficients, III

Calibration equation, I

Channel (of a radiometer), III

Circular polarization, III

Close-couple, II

Coherency matrix, III

Coherent detection, III

Completely polarized wave, III

Completely unpolarized wave, III

Complex correlator, III

Complex cros-correlator, III

Complex degree of coherence, III

Corrected result, IVM, GUM

Correlator, III

Cross-correlator, III

Degradation factor, III

Degree of polarization, III

Delay line, III

Digital correlator, III

Digital cross-correlator, III

Direct correlating correlator, III

Direct correlating polarimetric radiometer, III

Directivity, II

Drift, I

Dwell time, II

Effective black-body brightness temperature, I

Effective noise bandwidth, II

Emissivity of surface, I

Error, I

Experimental standard deviation, I

Field of view

Effective field of view, II

Instantaneous field of view (IFOV), II

Static IFOV, II

Static IFOV-Cross Track, II

Static IFOV-Down Track, II

Fringe-washing effect, III

Fully polarimetric calibration, III

Fully polarimetric calibration standard, III 
Fully polarimetric radiometer, III

Gain, see Antenna gain, Receiver gain, System gain

Gain matrix, III

Horizontal brightness temperature, III

Horizontal polarization, III

Incoherent detection, III

Inter-channel cross-talk, III

Limiting radiometric resolution, II

Linear polarization, III

Linearly polarized calibration standard, III

Measurand, IVM, GUM

Modified Stokes parameters, III

Modified Stokes parameters in brightness temperature, III

Modified Stokes vector in brightness temperature, III

Noise-equivalent Bandwidth, II

Noise figure, II

Noise temperature, I; see also Receiver noise temperature, Reference temperature,

System noise temperature

(Normalized) antenna pattern, II

Offset vector, III

Operating noise figure, II

Partially polarized wave, III

Perfect blackbody, II

Phase modulation, III

Phase retardation plane, III

Pixel-to-pixel accuracy, II

Platform motion compensation, III

Poincaré sphere, III

Polarimetric brightness temperautres, III

Polarimetric calibration, III

Polarimetric calibration standard, III

Polarimetric channels, III

Polarimetric radiometer, III

Polarization, III

Polarization combining network, III

Polarization combining radiometer, III

Polarization frame, III

Polarized brightness temperature scene, III

Polarizing grid, III

Principal polarizations, III

Quantization level, I

Radiance, I

Radiation intensity, II

Radiometer, II

Radiometric resolution, II

Radiometric sensitivity, II 
Random error, I

Receiver gain, II

Receiver noise figure, II

Receiver noise temperature, II

Reference temperature, I

Relative error, I

Relative uncertainty, I

Repeatability, I

Reproducibility, I

Resolution, I

Limiting radiometric resolution, II

Radiometric resolution, II

Radiometric resolution per beam width, II

Spatial resolution, II

Response of a fully polarimetric radiometer, III

Response time, I

Result of a measurement, IVM, GUM

Sense of polarization, III

Sensitivity, I

Spectral brightness density, I

Spectral radiance density, I

Stability, I

Standard

Check standard, I

Measurement standard, IVM

Primary standard, IVM

Secondary standard, IVM

Reference standard, IVM

Working standard, IVM

Transfer standard, IVM

Stokes parameter mixing, III

Stokes parameters, III

Stokes parameters in brightness temperature, III

Stokes vector in brightness temperature, III

Systematic error, I

System gain, II

System noise figure, II

System noise temperature, II

Traceability, I

Tri-polarimetric calibration, III

Tri-polarimetric calibration standard, III

Tri-polarimetric radiometer, III

True value, I

Type-A uncertainty, I

Type-B uncertainty, I

Uncertainty budget, I 
Uncertainty of measurement, I

Unpolarized brightness temperature scene, III

Unpolarized calibration, III

Unpolarized calibration standard, III

Validation, I

Verification, I

Vertical brightness temperature, III

Vertical polarization, III 



\section{NIST Technical Publications}

\section{Periodical}

Journal of Research of the National Institute of Standards and Technology-Reports N1ST research and development in metrology and related fields of physical science, engineering, applied mathematics, statistics. biotechnology, and information technology. Papers cover a broad range of subjects, with major emphasis on measurement methodology and the basic technology underlying standardization. Also included from time to time are survey articles on topics closely related to the Institute's technical and scientific programs. Issued six times a year.

\section{Nonperiodicals}

Monographs-Major contributions to the technical literature on various subjects related to the Institute's scientific and technical activities.

Handbooks-Recommended codes of engineering and industrial practice (including safety codes) developed in cooperation with interested industries, professional organizations, and regulatory bodies.

Special Publications-Include proceedings of conferences sponsored by NIST, NIST annual reports, and other special publications appropriate to this grouping such as wall charts, pocket cards, and bibliographies.

National Standard Reference Data Series-Provides quantitative data on the physical and chemical properties of materials, compiled from the world's literature and critically evaluated. Developed under a worldwide program coordinated by NIST under the authority of the National Standard Data Act (Public Law 90 396). NOTE: The Journal of Physical and Chemical Reference Data (JPCRD) is published bimonthly for NIST by the American Institute of Physics (AIP). Subscription orders and renewals are available from AIP, P.O. Box 503284. St. Louis, MO 63150-3284.

Building Science Series-Disseminates technical information developed at the Institute on building materials, components, systems, and whole structures. The series presents research results, test methods, and performance criteria related to the structural and environmental functions and the durability and safety characteristics of building elements and systems.

Technical Notes-Studies or reports which are complete in themselves but restrictive in their treatment of a subject. Analogous to monographs but not so comprehensive in scope or definitive in treatment of the subject area. Often serve as a vehicle for final reports of work performed at NIST under the sponsorship of other government agencies. Voluntary Product Standards-Developed under procedures published by the Department of Commerce in Part 10 , Title 15, of the Code of Federal Regulations. The standards establish nationally recognized requirements for products. and provide all concerned interests with a basis for common understanding of the characteristics of the products. NIST administers this program in support of the efforts of private-sector standardizing organizations. Order the following NIST publications-FIPS and NISTIRs-from the National Technical Information Service, Springfield, VA 22161.

Federal Information Processing Standards Publications (FIPS PUB)-Publications in this series collectively constitute the Federal Information Processing Standards Register. The Register serves as the official source of information in the Federal Government regarding standards issued by NIST pursuant to the Federal Property and Administrative Services Act of 1949 as amended, Public Law 89-306 (79 Stat. 1127), and as implemented by Executive Order 11717 (38 FR 12315, dated May 11, 1973) and Part 6 of Title 15 CFR (Code of Federal Regulations).

NIST Interagency or Internal Reports (NISTIR)-The series includes interim or final reports on work performed by NIST for outside sponsors (both government and nongovernment). In general, initial distribution is handled by the sponsor; public distribution is handled by sales through the National Technical Information Service, Springfield, VA 22161, in hard copy, electronic media, or microfiche form. NISTIR's may also report results of NIST projects of transitory or limited interest, including those that will be published subsequently in more comprehensive form. 
U.S. Department of Commerce

National Bureau of Standards and Technology

325 Broadway

Boulder, CO 80305-3337

Official Business

Penalty for Private Use $\$ 300$ 